\title{
Who Donates Their Bodies to Science? The Combined Role of Gender and Migration Status Among California Whole-body Donors
}

\section{Citation}

Asad, Asad L., Michel Anteby, and Filiz Garip. "Who Donates Their Bodies to Science? The Combined Role of Gender and Migration Status Among California Whole-body Donors." Social Science \& Medicine 106 (April 2014): 53-58.

\section{Published Version}

http://www.sciencedirect.com/science/article/pii/S0277953614000689

\section{Permanent link}

http://nrs.harvard.edu/urn-3:HUL.InstRepos:12748548

\section{Terms of Use}

This article was downloaded from Harvard University's DASH repository, and is made available under the terms and conditions applicable to Open Access Policy Articles, as set forth at http:// nrs.harvard.edu/urn-3:HUL.InstRepos:dash.current.terms-of-use\#OAP

\section{Share Your Story}

The Harvard community has made this article openly available.

Please share how this access benefits you. Submit a story.

\section{Accessibility}


Who donates their bodies to science? The combined role of gender and migration status among California whole-body donors

\author{
Asad L. Asad \\ Michel Anteby \\ Filiz Garip
}

Harvard University

[Published in Social Science \& Medicine, 106 (2014), 53-58] 


\begin{abstract}
The number of human cadavers available for medical research and training, as well as organ transplantation, is limited. Researchers disagree about how to increase the number of whole-body bequeathals, citing a shortage of donations from the one group perceived as most likely to donate from attitudinal survey data - educated white males over 65 . This focus on survey data, however, suffers from two main limitations: First, it reveals little about individuals' actual registration or donation behavior. Second, past studies' reliance on average survey measures may have concealed variation within the donor population. To address this limitation, we employ cluster analysis on all whole-body donors' data from the Universities of California at Davis, Irvine, Los Angeles, and San Francisco. Two donor groups emerge from the analyses: One is made of slightly younger, educated, married individuals, an overwhelming portion of whom are U.S.-born and have U.S.-born parents, while the second includes mostly older, separated women with some college education, a relatively higher share of whom are foreign-born and have foreign-born parents. Our results demonstrate the presence of additional donor groups within and beyond the group of educated and elderly white males previously assumed to be most likely to donate. More broadly, our results suggest how the intersectional nature of donors' demographics - in particular, gender and migration status - shapes the configuration of the donor pool, signaling new ways to possibly increase donations.
\end{abstract}

Keywords: United States; whole-body donations; cluster analysis; migration status; gender 


\section{Who Donates Their Bodies to Science? The Combined Role of Gender and Migration Status among California Whole-Body Donors}

\section{Introduction}

The importance of anatomical gifts - the donation of all or part of a human body for the purpose of research, education, or transplantation - is indisputable. Physicians and medical professionals in these fields rely almost exclusively on voluntary whole-body donations to provide sufficient numbers of cadavers, but demand often far outpaces supply (Agthong \& Wiwanitkit, 2002; Anteby \& Hyman, 2008; Dasgupta, 2004). With an estimated 18,000 cadaver donations per year in the United States (Becker \& Elias, 2007), and advances in surgical procedures that require cadaveric testing, scholarly and professional attention has turned to uncovering the sociodemographic factors that predict whether an individual will donate all or part of his or her body to science upon death.

Past work - focusing on characteristics such as age, race, marital status, and education - has employed regression analyses on attitudinal survey data to uncover the groups, on average, most likely to donate their bodies to science. These studies have found that educated, married, and white males over the age of 65 are more likely than other sociodemographic groups to bequeath their bodies (Boulware et al., 2004; Dluzen et al., 1996; Harrington \& Sayre, 2006; Healy, 2004; Sanner, 2006). As a result, researchers have suggested that recruitment efforts focus on this uniform group. Despite its large size and its potentially heterogeneous composition with respect to other demographic indicators, scholars and practitioners alike have argued that this population represents the best opportunity for securing whole-body donations. In their attempt to remedy cadaver shortages, then, donation programs have adjusted the methods and efficacy of their donor recruitment, with some going so far as to send nursing homes an informational video encouraging whole-body registration and donation (Dasgupta, 2004).

One method for increasing the number of cadaveric donations is through targeting sociodemographic groups beyond those identified by attitudinal measures as the most likely to donate - that is, elderly, educated, and married white males. In particular, despite several studies investigating the importance of gender (Sanner, 2006) and, to a lesser extent, migration status (Salim et al., 2010), past work has not fully examined the role of women and immigrants in the donation process. Women, for example, have been described as amenable to donating certain organs, but also have been characterized as reluctant to actually volunteer their bodies to science (Sanner, 1994, 2006). Similarly, how migration status - that is, whether an individual is a firstor second-generation migrant - influences donation outcomes has been underexplored. Some work internationally has identified first- and second-generation immigrants as a feasible pool for increasing the quality of blood donations (Grassineau et al., 2007), but to our knowledge, no work has evaluated this finding with respect to anatomical donation in the United States. Indeed, a growing body of literature suggests that first- and second-generation migrants may differentially experience health and health care when compared to the native-born (AbraidoLanza et al., 1999; Viruell-Fuentes, 2007). As such, one might expect differences in donor profiles by migration status. 
In brief, past studies' emphasis on the willingness of elderly, educated, and white males to donate their bodies to science, while shedding great insights into assumed contemporary best strategies for recruiting anatomical donors, leaves unanswered two important questions: First, what does attitudinal data on willingness to donate reveal about individuals' actual registration or donation behavior? Second, using this same survey data, studies to date have sought to identify the "average" donor. Nevertheless, relying on averages alone may conceal the variation within the donor population. Are there additional groups of donors with different configurations of characteristics that donate their bodies to science? If so, these previously undiscovered groups may add to our understanding of donation dynamics and be more effectively targeted than the assumed "most-likely" donor group identified by past work. The goal of this study is thus to investigate and test for the presence of additional groups of whole-body donors both within and beyond the group of elderly, educated, and white males depicted in past work. By identifying new donor types, this study suggests previously overlooked donation dynamics - particularly with respect to gender and migration status - and ways to possibly recruit additional whole-body donors, whose anatomical gifts are critical for medical research and training, as well as organ transplantation.

\section{Methods}

\section{Study Setting}

We selected the University of California system, as it collectively registers more than 1,000 anatomical donors each year. We collected our data from the Universities of California at Davis, Irvine, Los Angeles, and San Francisco (UCD, UCI, UCLA, and UCSF, respectively), which support whole-body donations through their medical schools. Since California law requires that any whole-body registrants be permanent residents of the state, and since the UC system secures most of its donations in-state, this site provides unique insight into who chooses to become a whole-body donor in California. Beyond non-residents' registration being highly unlikely, the data set allows us to maximize the number of valid observations within one particular geographic region. Though the resulting sample may not be representative of the national population of whole-body donors, this study is primarily concerned with the identification of new donor types. If more donor groups than previously hypothesized to exist are revealed, our analyses may be suggestive of additional donor groups and unexplored donation dynamics in other locales.

\section{Data}

We compiled records on individuals who registered for the whole-body donation program at the UCD, UCI, UCLA, and UCSF campuses after September 2008, when all campuses converted to a unified digital data collection system. We included in the data set only actual donors - that is, deceased registrants, for whom the records were more complete. Examining actual donors provide a more accurate view of donations than studying registrants alone since, in some cases, registrants' families may be reluctant to follow through with the donation. Registration data thus fail to capture actual instances of donation. The final data set contains observations from 1,356 donors who died prior to June 2011, when we received the data. We dropped the cases that lacked information on the sociodemographic attributes of interest: age (23 out of 1,356), education (60 out of the remaining 1,333), marital status (10 out of 1,273), race (1 out of 1,263), 
migrant status (15 out of 1,262), and parents' migrant status (90 out of 1,247). The resulting data set has 1,157 observations - $254(22 \%)$ from UCD, 142 (12\%) from UCI, 216 (19\%) from UCLA, and 545 (47\%) from UCSF.

\section{Data Collection}

Although each campus administers its own program, the University of California system has standardized the registration procedure for whole-body donation. Potential donors independently request information (by phone, by mail, or online), complete a registration packet, and return the signed forms indicating their intent to donate their bodies after death to the relevant institution. Each donor must complete a separate registration form. Records include each registrant's vital statistics, including full name, year of birth, registration date, sex, race/ethnicity, religious affiliation, and marital status. Registrants are also asked to provide the names and birthplace of their father and mother, as well as information on legal next of kin. Upon a registrant's death, administrators with the University of California whole-body programs contact legal next of kin, verify the information on the registration form, and update any missing information.

Access to the data was granted by the program director after the University of California and Harvard University institutional review boards' approvals. The institutional review boards waived the need for written informed consent from participants in light of Title 45, part 46, section 116 (d) in the U.S. Code of Federal Regulations. Deleting registrants' names following coding of donor relationships ensured confidentiality.

\section{Analysis}

Cluster analysis was used to discover groups with similar attributes in the data. Six attributes at the time of registration were selected to partition the data: (1) age, (2) sex, (3) marital status (indicators for whether individual is married or separated), (4) race (white, black, or other Hispanic or Asian), (5) education (high school, some college, college, or advanced degree), and (6) migrant status (indicators of whether individual is foreign-born or has a parent that is foreignborn).

Clustering methods are typically sensitive to scaling of attributes, which determines the importance assigned to a particular attribute. To avoid an arbitrary weighting, the only nonbinary attribute, age, was dichotomized such that the values above the median age are converted to 1 and those below it to 0 .

Employing the popular K-means method as implemented in Matlab software (version 8.0) (Matlab, 2011), the classical algorithm divides the data into a given number of groups ("clusters") based on selected attributes so that the cases in a group are as similar as possible. This standard algorithm iterates between computing K cluster centroids by minimizing the within cluster variance and updating cluster memberships (Hastie et al., 2009). The K-means algorithm is typically very fast, but sometimes gets stuck at local minima, which may prevent the discovery of the optimal clustering solution in the data. To overcome this problem, the algorithm is run 1000 times in Matlab, each with a random initialization, and the solution with the minimum within-cluster variance is selected as the optimal solution. The output is a cluster membership for 
each observation and a centroid for each cluster that represents the average of the cases in that cluster.

The method requires the identification of a measure of similarity to assess how "close" cases are to one another in the attribute space. Using city block distance aptly deals with binary data and reflects the authors' substantive preference to treat two individuals who share a trait (e.g., low education) as equally similar to one another as two individuals who both lack the trait. For every pair of individuals $i$ and $j$, the city block distance, $d_{i j}$, is the sum of the absolute differences in the values $x_{i l}$ and $x_{j l}$ of each attribute $l=1, \ldots, p$,

$$
d_{i j}=\left(\sum_{l=1}^{p}\left|x_{i l}-x_{j l}\right|\right)
$$

The method also requires the researcher to supply the number of clusters, $\mathrm{K}$, to the K-means algorithm. By construction, this algorithm locates K clusters even when no such structure exists in the data. To avoid obtaining artificial partitions, researchers use cluster validation measures to choose the optimal number of clusters. This process is similar to model selection in regression analysis, where researchers use the likelihood ratio, or another criterion, to select the best and most parsimonious model for the data. This study uses four cluster validation measures to estimate the number of clusters in the data. These measures are implemented in the clValid and fpc packages in R software (Brock et al., 2008; Hennig, 2010; R Development Core Team, 2012).

\section{[Figure 1. Cluster Validation Measures Across Number of Clusters]}

The two panels in Figure 1 present two measures plotted against the number of clusters ranging from two to six. For the average silhouette distance in the left-hand panel, higher values indicate higher cluster quality. For the connectivity index in the right-hand panel, lower values indicate higher cluster quality. Both measures obtain their optimal value for the 2-cluster solution.

\section{[Figure 2. Cluster Stability Measures Across Number of Clusters]}

Two additional measures, plotted against the number of clusters in Figure 2, capture the "stability" of clusters to changes in the attribute space. Specifically, the average proportion of non-overlap (APN) in the left-hand panel, and the average distance between means (ADM) in the right-hand panel, both evaluate whether the clustering solution remains stable if attributes are removed one at a time. For both measures, lower values indicate more stable clustering solutions. The 2-cluster solution yields the best score in both cases.

Based on these results, the 2-cluster solution is selected, which is optimal for all four measures.

\section{Results}

The three columns in Table 1 present the mean values of attributes in the overall data as well as in each of the two clusters. The last two rows show the number and percentage of individuals in 
each cluster, which appear to be relatively uniform. The attributes are measured at the time of individuals' registration to donate their bodies.

\section{[Table 1. Individual Characteristics by Cluster Membership]}

The two clusters differ significantly $(p<0.05)$ in nine of the thirteen attributes. Compared to the second cluster, the first cluster contains slightly younger donors (average age 71.4 vs. 80.5); a higher share of men ( 0.78 vs. 0.26$)$; a higher share of married individuals (0.76 vs. 0.03$)$; a higher share of college ( 0.28 vs. 0.18$)$ and advanced degree ( 0.17 vs. 0.13$)$ holders; a lower share of foreign-born individuals ( 0.09 vs. 0.15$)$, and a lower share of individuals with foreign-born parents $(0.27$ vs. 0.37$)$.

We perform two robustness checks. First, if geography is important when considering who donates, we might expect the foreign-born donors to cluster in the two Southern California campuses, Irvine and Los Angeles. To test this intuition, we assess if the clusters differ in their presence across the four campuses included in the study. Difference-of-means test suggest that the two clusters do not differ significantly in their presence in any of the four campuses $(\mathrm{p}<0.05)$. Thus, our results are not driven by the potential geographic differences in migrant composition. These analyses are available upon request.

Second, we assess whether our results are driven by the fact that women, on average, live longer than men. If so, does the second cluster - consisting of a larger share of separated (i.e., widowed or divorced) older women - and the first cluster - containing a larger share of relatively younger and married men - reflect couples donating together? We first test this intuition descriptively, finding only $55 \%$ of the individuals in the second cluster to be widowed; the rest are divorced. To further test this idea, we match our donors of opposite sexes on their last names and zip codes to identify potential co-donating couples. We find that less than 7-percent of individuals in the entire sample fit into this category. It is unlikely that our clusters reflect a simple division between husbands (cluster 1) and wives (cluster 2).

Thus, cluster one consists of slightly younger, educated, married individuals, an overwhelming portion of whom are U.S.-born and have U.S.-born parents, while cluster two includes mostly older, separated women (i.e., widowed or divorced) with some college education, a relatively higher share of whom are foreign-born and have foreign-born parents.

\section{Discussion}

Physicians and other medical professionals rely on the availability of cadavers for research and training purposes, as well as transplantation. Indeed, without uncovering new groups from which to cull donations, "it will be impossible for the pupils to learn anatomy, and without anatomy, neither surgeons nor physicians can practice with the least prospect of benefitting their patients" (Dasgupta, 2004). Understanding that demand outpaces supply, we sought to identify additional whole-body donor groups beyond those primarily shown to exist in current research - namely, educated and married white males over the age of 65 . 
The few studies that rigorously test what types of individuals are likely to donate their bodies to science have up to now been unanimous in concluding that elderly, educated, married, and white men represent an important pool of donors (Anteby et al., 2012; Boulware et al., 2004; Dluzen et al., 1996; Harrington \& Sayre, 2006). The first cluster corroborates this observation. Although its average age of 71.4 years is higher than the average 65 observed in other work, this could result from our analysis of actual (deceased) donors rather than living registrants. The vast majority of past studies have only described the sociodemographic characteristics of living registrants. The limited work focused on actual donations provides only descriptive analyses of the average donor profile and does not examine distinct configurations of donor groups (i.e., variation within the donor pool) (Dluzen et al., 1996). Furthermore, they could not account for the possibility that registrants' bodies were ultimately not bequeathed to science. Indeed, the literature suggests a number of possibilities that may exacerbate the cadaver shortage beyond a lack of registration, such as the attitudes of kinship networks toward body donation (Agthong \& Wiwanitkit, 2002). Even if the deceased agrees to donate their corpus pre-mortem, next of kin may renege on the decedent's commitment. Our focus on actual instances of donation, then, allows us to circumvent this limitation.

In contrast to the first cluster, the second includes mostly older, separated women with some college education, a relatively higher share of whom are foreign-born and have foreign-born parents. This second group breaks from past work by demonstrating heterogeneity within the donor population. While elderly, educated, and married white males represent the group most amenable to whole-body donation, on average, this emphasis on averages has concealed the presence of at least one additional donor group - in this case, women who are more likely to be foreign-born and have foreign-born parents. To our knowledge, the second cluster represents a unique intersection of sociodemographic characteristics that past work has associated with anatomical donations. This cluster therefore highlights the need to pay closer attention to the relationships between multiple dimensions of individuals' social relationships and subject formations, or "interesectionality" (Crenshaw, 1991; McCall, 2005), when analyzing who donates his or her body to science.

Past work has shown women to be amenable to blood (Piliavin, 2003) and oocyte donation (Almeling, 2011; Kenney \& McGowan, 2010), but has depicted the population as reluctant to actually volunteer their bodies to science (Sanner, 1994, 2006). Attempts to explain this disconnect have yielded mixed results. Studies have reported men and women's contrasting attitudes toward whole-body donation, with men demonstrating greater willingness to donate than women (Boulware et al., 2004). This differential might be due to relative discomfort associated with performing medical procedures on dead bodies (Sanner, 1994). Registering to donate with one's spouse, however, increases the likelihood of women's whole-body donation (Anteby et al., 2012). Although the mechanisms underlying this decision-making process are unclear, some have suggested that the finality of the donation decision "may represent a feeling of closure" for both partners (Lagwinski et al., 1998: 260). Overall, women are widely assumed to be reluctant to donate their bodies to science, but our results suggest this is not always the case. By considering the intersection of donors' demographics (e.g., gender and/or marital status and/or migration status), our findings suggest a more complex view of donation dynamics than previously depicted in the literature. 
Our findings also inform literatures on immigration and medicine by emphasizing the role of migration status in the donation decision. Research in both fields finds that being an immigrant or the child of one can lead to differential integration outcomes across several dimensions, including health (Abraido-Lanza et al., 1999; Viruell-Fuentes, 2007), education (Kao \& Tienda, 1995; Suárez-Orozco \& Suárez-Orozco, 2009), and overall life chances (Kasinitz et al., 2008; Portes \& Rumbaut, 2006). Including parents' and donors' migration status in our analyses revealed the attributes' importance in discerning new configurations of donors, an occurrence previously untested in the U.S. context. This finding, however, adds to a growing line of research conducted internationally that identifies first- and second-generation immigrants as an important pool for increasing the diversity and quality of blood donations (Grassineau et al., 2007). Others, using a representative random sample of migrants in Spain, find similar results for organ donation, with female migrants reporting more comfort with donating than men (López et al., 2012). In-line with past work, then, the second cluster extends the literatures on both gender and migration by uncovering a unique intersectional configuration of whole-body donors in the U.S. context who may be more actively recruited by procurement agencies.

\section{Future Directions for Research}

Social scientists have long recognized the importance of typologies for providing conceptual frameworks for testing hypotheses (Lazarsfeld, 1937; Weber, [1922] 1978). The typology presented here suggests several avenues for future work: First, how should recruitment strategies vary by donor group? Second, how might we capitalize on social networks to bolster the recruitment process? We briefly address each of these questions in turn.

\section{How Should Recruitment Strategies Vary by Donor Types?}

Current recruitment strategies for additional whole-body donors primarily have targeted those groups assumed, up to now, to constitute the most likely donors. In the same way that surgeons acquire distinct classification schemes during their training to account for medical errors and inform future behavior (Bosk, 1979), whole-body program administrators, over time, might have developed classification understandings and assumptions about the ideal donor type (namely, educated and elderly white males), which implicitly guide their recruitment efforts. Our results suggest, however, a need to go beyond that ideal type, and consider the variation that exists within and beyond that category of donors. More specifically, our study highlights the need to pay more attention to donors' intersectional demographic attributes. Future research should thus focus on uncovering how layered and intersecting attributes, such as gender and migration status, might play out in the decision to donate one's body to science.

Qualitative work would be especially useful here, though the sensitive nature of the topic may make it difficult for researchers to determine the real-time decision-making processes through which an individual chooses to register to be a donor and, ultimately, her or his family's decision to follow through with her or his wishes or not. Past interview-based studies have suggested that people make the decision to donate with great difficulty and with impressive variation in the reasons they give for having donated or not (Dixon-Woods et al., 2008). If future studies can shed light on these questions with respect to distinct intersectional configurations of donor groups, then procurement agencies can concertedly recruit the self-selected groups more 
effectively. In this way, we would expect that both registrations and, ultimately, donations to cadaver procurement programs would increase.

\section{Can Social Networks Help?}

Past work has shown that registered whole-body donors report knowing someone intending to donate, with up to $92 \%$ of people sharing their donation status with their loved ones (Richardson \& Hurwitz, 1995). In such a case, our findings may inform future work on the role of social networks in the recruitment of whole-body donors. The characteristics that distinguish the two donor groups we describe above (e.g., gender, migration status, and educational attainment) are those that also stratify social networks (DiMaggio \& Garip, 2011). In particular, homophily - the tendency of persons to form networks with socioeconomically and demographically similar peers - suggests that more targeted recruitment efforts customized to these groups would create faster diffusion through social networks and lead to higher rates of adoption of the practice (i.e., donation) (Centola, 2011; see DiMaggio \& Garip, 2012 for a review). Thus, we argue that more targeted recruitment efforts tailored to these groups would increase the number of whole-body registrants and, potentially, donors through higher rates of diffusion through social networks.

\section{Conclusion}

Using data on actual whole-body donors in California, the current analysis suggests the existence of two whole-body donor groups. Relative to the second, the first consists of younger, educated, and married individuals who are more likely to be U.S.-born and have U.S.-born parents. In contrast, the second includes mostly older, separated women with some college education, a relatively higher share of whom are foreign-born and have foreign-born parents. The presence of these two groups both corroborates and extends the findings of past work. We confirm the presence of the "average" donor base of elderly, educated, and married white men in a sample of donors, while simultaneously uncovering a unique group of women and migrants that previous work has overlooked.

These findings are particularly pertinent at a time when the shortage of anatomical donations is reaching critical mass (Agthong \& Wiwanitkit, 2002; Anteby \& Hyman, 2008; Dasgupta, 2004). The situation is especially dire for those awaiting organ transplants. With approximately 100,000 patients in need of a new organ and $25 \%$ of this group dying before receiving one, the identification of new donor groups may represent a fruitful opportunity for increasing the number of organ transplantations (Salim et al., 2010; Zawistowski \& DeVita, 2003). Previously, organs from the cadavers of elderly patients had only been used for living-related transplants (Zawistowski \& DeVita, 2003). In recent years, however, studies have shown that anatomical donations obtained from elderly living-unrelated donors achieve impressive success rates (Andrés et al., 2009; First, 2001; Zawistowski \& DeVita, 2003). Indeed, Spain - which maintains a less age-restrictive anatomical gifting program than its Western counterparts - reports an 18.3percent higher rate of successful organ transplantations than the United States due to its reliance on elderly donors (Cuende et al., 2007). Thus, the ability to recruit anatomical donations from a more expansive pool of donors - those identified by the two-cluster solution presented above has tremendous potential to facilitate life-saving transplantation, in addition to supporting medical research and education. 
In brief, the typology we present here suggests that we must revisit past assumptions on who chooses to donate and why. Using these findings as a starting point, future work should investigate how the intersectional nature of individuals' demographic attributes might impact donation behavior, with special attention paid to how the intersection of gender and migration status might play into these donation dynamics. In so doing, procurement efforts can be refocused to maximize whole-body donor registrations and, ultimately, increase the supply of cadavers used for transplantation and medical research and training. 


\section{References}

Abraido-Lanza, A.F., Dohrenwend, B.P., Ng-Mak, D.S., \& Turner, J.B. (1999). The Latino mortality paradox: a test of the" salmon bias" and healthy migrant hypotheses. American Journal of Public Health, 89, 1543-1548.

Agthong, S., \& Wiwanitkit, V. (2002). Cadaver Donation: A Retrospective Review at the King Chulalongkorn Memorial Hospital, Bangkok. Southeast Asian Journal of Tropical Medicine and Public Health, 33, 166-167.

Almeling, R. (2011). Sex cells: The medical market for eggs and sperm: Univ of California Press.

Andrés, A., Polanco, N., Cebrian, M.P., Sol Vereda, M., Nuño, E., Bello, T., et al. (2009). Kidneys from Elderly Deceased Donors Discarded for Transplantation. Transplanation Proceedings, 41, 2379-2381.

Anteby, M., Garip, F., Martorana, P.V., \& Lozanoff, S. (2012). Individuals' Decision to Co-Donate or Donate Alone: An Archival Study of Married Whole Body Donors in Hawaii. PLoS One, 7, 1-6.

Anteby, M., \& Hyman, M. (2008). Entrepreneurial Ventures and Whole-Body Donations: A Regional Perspective from the United States. Social Science \& Medicine, 68, 963-969.

Becker, G.S., \& Elias, J.J. (2007). Introducing Incentives in the Market for Live and Cadaveric Organ Donations. Journal of Economic Perspectives, 21, 3-24.

Bosk, C.L. (1979). Forgive and remember: managing medical failure: University of Chicago Press.

Boulware, L.E., Ratner, L.E., Cooper, L.A., LaVeist, T.A., \& Pow, N.R. (2004). Whole Body Donation for Medical Science: A Population-Based Study. Clinical Anatomy, 17, 570-577.

Brock, G., Pihur, V., Datta, S., \& Datta, S. (2008). clValid: An R Package for Cluster Validation. Journal of Statistical Software, 25, 1-22.

Centola, D. (2011). An experimental study of homophily in the adoption of health behavior. Science, 334, 1269-1272.

Crenshaw, K. (1991). Mapping the margins: Intersectionality, identity politics, and violence against women of color. Stanford law review, 1241-1299.

Cuende, N., Cuende, J.I., Fajardo, J., Huet, J., \& Alonso, M. (2007). Effect of Population Aging on the International Organ Donation Rates and the Effectiveness of the Donation Process. American Journal of Transplanation, 7, 1526-1535.

Dasgupta, N. (2004). Unclaimed Bodies at the Anatomy Table. Journal of the American Medical Association, 291, 122.

DiMaggio, P., \& Garip, F. (2011). How network externalities can exacerbate intergroup inequality. American Journal of Sociology, 116, 1887-1933.

DiMaggio, P., \& Garip, F. (2012). Network effects and social inequality. Annual Review of Sociology, 38, 93-118.

Dixon-Woods, M., Cavers, D., Jackson, C.J., Young, B., Forster, J., Heney, D., et al. (2008). Tissue Samples as 'Gifts' for Medical Research: A Qualitative Study of Families and Professionals. Medical Law Journal, 9, 131-150.

Dluzen, D.E., Brammer, C.M., Bernard, J.C., \& Keyser, M.L. (1996). Survey of Cadveric Donors to a Body Donation Program: 1978-1993. Clinical Anatomy, 9, 183-192. 
First, M.R. (2001). The Organ Shortage and Allocation Issues. Transplantion Proceedings, 33, 806-810.

Grassineau, D., Papa, K., Ducourneau, A., Duboz, P., Boëtsch, G., \& Chiaroni, J. (2007). Improving minority blood donation: anthropologic approach in a migrant community. Transfusion, 47, 402-409.

Handl, J., Knowles, J., \& Kell, D.B. (2005). Computational cluster validation in postgenomic data analysis. Bioinformatics, 21, 3201-3212.

Harrington, D.E., \& Sayre, E.A. (2006). Paying for Bodies, But Not for Organs. Regulation, 29, 14-19.

Hastie, T., Tibshirani, R., \& Friedman, J. (2009). The Elements of Statistical Learning: Data Mining, Inference, and Prediction: Springer.

Healy, K. (2004). Altruism as an Organizational Problem: The Case of Organ Procurement. American Sociological Review, 69, 387-404.

Hennig, C. (2010). fpc: Flexible Procedures for Clustering. Rpackage Version 2.0-3.

Kao, G., \& Tienda, M. (1995). Optimism and achievement: The educational performance of immigrant youth. Social science quarterly, 76, 1-19.

Kasinitz, P., Mollenkopf, J.H., Waters, M.C., \& Holdaway, J. (2008). Inheriting the city: The children of immigrants come of age: Russell Sage Foundation.

Kenney, N.J., \& McGowan, M.L. (2010). Looking back: egg donors' retrospective evaluations of their motivations, expectations, and experiences during their first donation cycle. Fertility and sterility, 93, 455-466.

Lagwinski, M., Bernard, J.C., Keyser, M.L., \& Dluzen, D.E. (1998). Survey of cadaveric donor application files: 1978-1993. Clinical Anatomy, 11, 253-262.

Lazarsfeld, P.F. (1937). Some remarks on the typological procedures in social research. Zeitschrift für Sozialforschung, 6, 119-139.

López, J.S., Valentín, M.O., Scandroglio, B., Coll, E., Martín, M.J., Sagredo, E., et al. (2012). Factors related to attitudes toward organ donation after death in the immigrant population in Spain. Clinical Transplantation, 26, E200-E212.

Matlab (2011). Matlab 2011a: The Language of Technical Computing. The Mathworks.

McCall, L. (2005). The complexity of intersectionality. Signs, 30, 1771-1800.

Piliavin, J.A. (2003). Why Do They Give the Gift of Life? A Review of Research on Blood Donors Since 1977. Transfusion, 30, 444-459.

Portes, A., \& Rumbaut, R.G. (2006). Immigrant America: a portrait: Univ of California Press.

Richardson, R., \& Hurwitz, B. (1995). Donors' Attitudes Towards Body Donation for Dissection. Lancet, 346, 277-280.

Rousseeuw, P.J. (1987). Silhouettes: A Graphical Aid to the Interpretation and Validation of Cluster Analysis. Computational and Applied Mathematics, 20, 53-65.

Salim, A., Berry, C., Ley, E.J., Schulman, D., Desai, C., Navarro, S., et al. (2010). The impact of race on organ donation rates in Southern California. Journal of the American College of Surgeons, 211, 596-600.

Sanner, M. (1994). A Comparison of Public Attitudes Toward Autopsy, Organ Donation, and Anatomic Dissection: A Swedish Survey. Journal of the American Medical Association, 271, 284-288.

Sanner, M. (2006). People's Attitudes and Reactions to Organ Donation. Mortality, 11, 133-150. 
Suárez-Orozco, C., \& Suárez-Orozco, M.M. (2009). Children of immigration: Harvard University Press.

Team, R.D.C. (2012). R: A language and Environment for Statistical Computing.

Viruell-Fuentes, E.A. (2007). Beyond acculturation: immigration, discrimination, and health research among Mexicans in the United States. Social Science \& Medicine, $65,1524-1535$.

Weber, M. ([1922] 1978). Economy and society: An outline of interpretative sociology: Univ of California Press.

Zawistowski, C.A., \& DeVita, M.A. (2003). Non-Heartbeating Organ Donation: A Review. Journal of Intensive Care Medicine, 18, 189-197. 
Table 1. Individual Characteristics by Cluster Membership

\begin{tabular}{|c|c|c|c|c|}
\hline & $\begin{array}{l}\text { Overall } \\
\text { Sample }\end{array}$ & Cluster 1 & Cluster 2 & \\
\hline Age & 76.4 & 71.4 & 80.5 & $*$ \\
\hline Male & 0.50 & 0.78 & 0.26 & $*$ \\
\hline Married & 0.36 & 0.76 & 0.03 & $*$ \\
\hline Separated (widowed or divorced) & 0.54 & 0.10 & 0.89 & * \\
\hline White & 0.90 & 0.89 & 0.90 & \\
\hline Black & 0.04 & 0.04 & 0.04 & \\
\hline Other (Hispanic or Asian) & 0.07 & 0.07 & 0.07 & \\
\hline High school & 0.27 & 0.25 & 0.28 & \\
\hline Some college & 0.26 & 0.22 & 0.29 & $*$ \\
\hline College & 0.22 & 0.28 & 0.18 & $*$ \\
\hline Advanced degree & 0.15 & 0.17 & 0.13 & $*$ \\
\hline Individual is a migrant (foreign-born)? & 0.12 & 0.09 & 0.15 & $*$ \\
\hline Individual has a migrant (foreign-born) parent? & 0.32 & 0.27 & 0.37 & $*$ \\
\hline $\mathrm{N}$ & 1,157 & 519 & 638 & \\
\hline (\% of total) & & $45 \%$ & $55 \%$ & \\
\hline
\end{tabular}


Figure 1. Cluster Validation Measures Across Number of Clusters
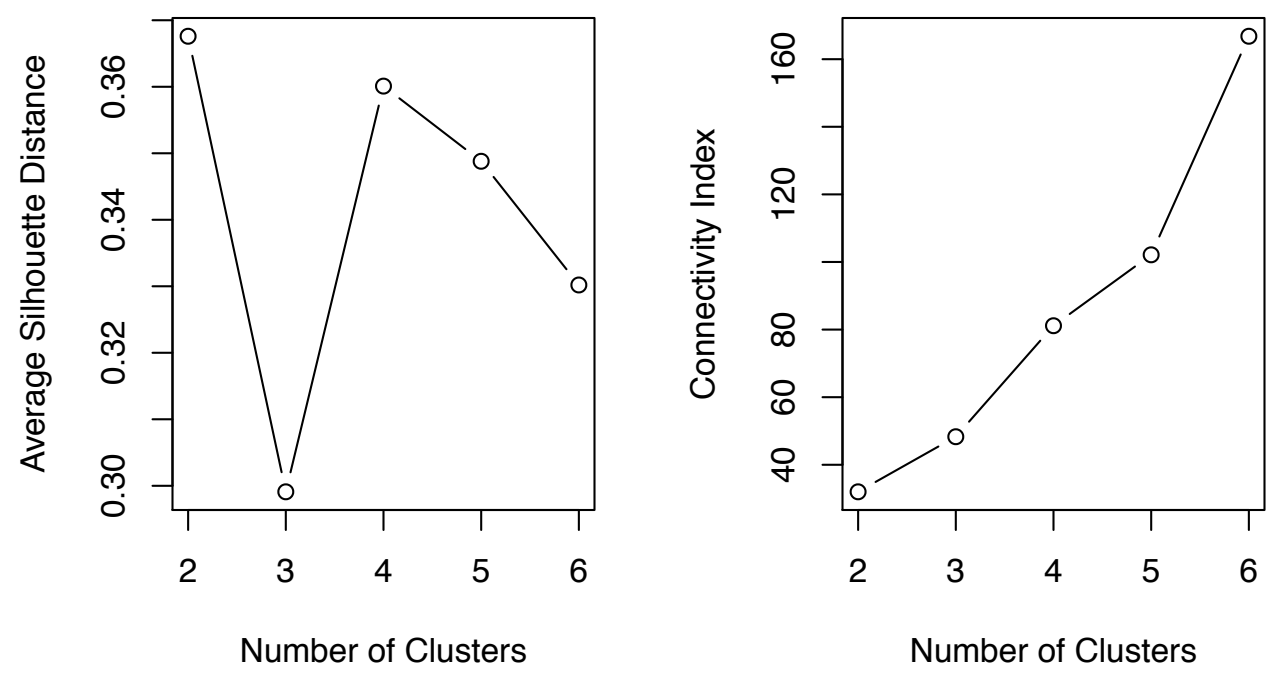

The average silhouette distance is the difference between the average distance between individuals in the same cluster and the smallest average distance to other clusters. ${ }^{1}$ The connectivity index counts the cases where observations are placed in different clusters than their nearest neighbors in the data space. ${ }^{2}$ For the former measure, higher values indicate higher cluster quality, while for the latter, lower values indicate higher cluster quality. 
Figure 2. Cluster Stability Measures Across Number of Clusters
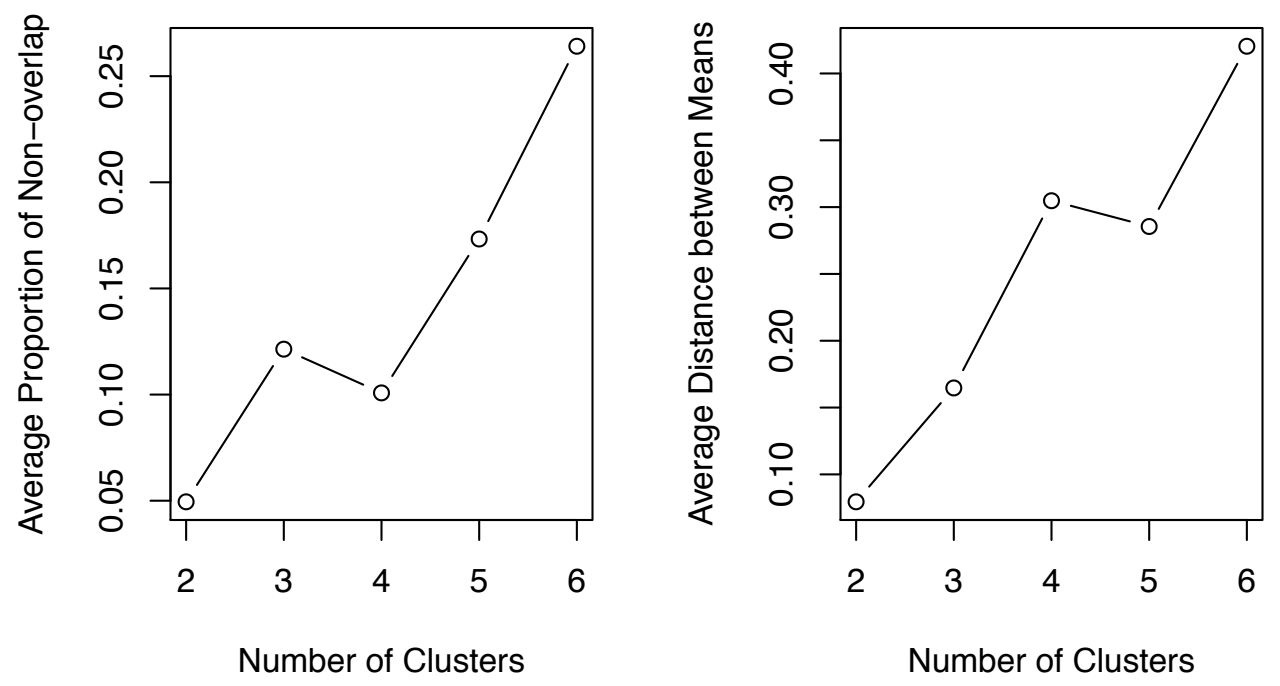

The average proportion of non-overlap computes the average proportion of cases that are not put in the same cluster if the attributes were deleted one at a time. The average distance between means computes the average distance between the mean attributes in the same cluster if the attributes were deleted one at a time. For both measures, lower values indicate more stable clustering solutions. 